\title{
Diffuse Anterior Retinoblastoma with Sarcoidosis-Like Nodule
}

\author{
Koji Kitazawaa, b, d Kenji Nagata ${ }^{a}$ Yukito Yamanaka ${ }^{a}$ \\ Yasumichi Kuwahara ${ }^{c}$ Tomoko Iehara $^{c}$ Shigeru Kinoshita ${ }^{b}$ \\ Chie Sotozono ${ }^{a}$ \\ Departments of ${ }^{\mathrm{a}}$ Ophthalmology, ${ }^{\mathrm{b}}$ Frontier Medical Technology for Ophthalmology and \\ ${ }^{c}$ Pediatrics, Kyoto Prefectural University of Medicine, and ${ }^{d}$ Baptist Yamasaki Eye Clinic, \\ Kyoto, Japan
}

\section{Key Words}

Retinoblastoma - Diffuse anterior retinoblastoma - Sarcoidosis · Diffuse infiltrating retinoblastoma - Enucleation · Chemotherapy · Anterior segment optical coherence tomography

\begin{abstract}
Background: Retinoblastomas account for $4 \%$ of malignancies in children, $1-2 \%$ of which are diffuse infiltrating retinoblastomas. Diffuse anterior retinoblastoma is rare and does not involve the retina. Here, we report on a diffuse anterior retinoblastoma with large sarcoidosislike nodules on the iris that were responsive to anti-inflammatory therapy. Case: We present a 6-year-old girl who had anterior uveitis with white nodules on the iris and posterior surface of the cornea in her right eye. The nodules initially responded well to anti-inflammatory treatment. However, anterior segment optical coherence tomography (AS-OCT) showed that the nodules gradually grew, shrinking the iris. We then collected the aqueous humor for diagnosis. A biopsy revealed clusters of small cells with a high nuclear-to-cytoplasm ratio with partial rosette formation. Therefore, we diagnosed diffuse anterior retinoblastoma without retinal involvement and performed enucleation of the right eye. The histopathology demonstrated undifferentiated cells similar to those seen on the biopsy, and tumor cells invaded the iris stroma, posterior surface of the cornea, ciliary body, and sclera. After the enucleation, she underwent chemotherapy and remains alive. Conclusion: A differential diagnosis of retinoblastoma should be considered when white nodules refractory to anti-inflammatory therapy occur in the eye, even in the absence of obvious retinal masses. AS-OCT findings are useful in assessing retinoblastoma.


Kitazawa et al.: Diffuse Anterior Retinoblastoma with Sarcoidosis-Like Nodule

\section{Introduction}

Retinoblastomas account for $4 \%$ of malignancies in children, and $95 \%$ of cases are detected before the age of 5 years [1]. RB1 gene mutations have been identified as a causative factor for retinoblastoma. Typical B-scan ultrasonography findings include high-density white retinal masses with an acoustic shadow. In some cases, retinoblastoma grows horizontally in the retina and is often mistaken for uveitis or inflammatory processes [2].

Herein, we report the case of a 6-year-old girl with anterior retinoblastoma with sarcoidosis-like nodules on the iris, without retinal involvement. Tumor progression was observed with anterior segment optical coherence tomography (AS-OCT). B-scan ultrasonography did not detect nodule calcification, but the nodules were initially responsive to antiinflammatory therapy.

\section{Case Report}

A 6-year-old girl with anterior uveitis and white nodules on the iris surface, who was not aware of any visual disturbance, attended the University Hospital, Kyoto Prefectural University of Medicine, in November 2013. There was no family history of eye disease. At the initial examination, her visual acuity was $20 / 20$, and the intraocular pressure in both eyes was $21 \mathrm{~mm} \mathrm{Hg}$. A physical examination showed that she had good general health. Of the three white nodules in the right eye, one was located on the iris, and the others were found on the posterior surface of the cornea (fig. 1a). A fundus examination revealed that there was no vitreous inflammation, obvious retinal lesions, or other abnormal findings. B-scan ultrasonography did not reveal any nodule calcifications. Anti-toxocara immunoglobulin G antibodies were not detected. The patient was administered topical anti-inflammation treatment for the anterior uveitis and nodules. The nodules gradually decreased in size; however, by November 2014, those on the posterior surface of the cornea had spread horizontally, and AS-OCT showed a shrunken iris (fig. 1b, c). These changes could have been a result of tumor migration and invasion. In December 2014, after explaining the risk of metastasis to her parents, we obtained informed consent to collect the aqueous humor to determine the diagnosis. The biopsy revealed clusters of small cells with a high nuclear-to-cytoplasm ratio with partial rosette formation (fig. 2a). Computed tomography, magnetic resonance imaging, positron emission tomography, and gallium scintigraphy confirmed the absence of invasion outside the eyeball. Based on these findings, we diagnosed diffuse anterior retinoblastoma without retinal involvement and performed enucleation of the right eye. Histopathology showed undifferentiated cells similar to those seen on the biopsy. Tumor cells had infiltrated the iris stroma, posterior surface of the cornea, ciliary body, and sclera, but they were not detected in the retina (fig. $2 \mathrm{~b}$ ). Serial sections confirmed that there were no tumor cells outside the eyeball. After enucleation, she underwent 6 cycles of chemotherapy and remains alive without relapse.

\section{Discussion}

We present a case of diffuse anterior retinoblastoma with the nodules on the iris and the posterior surface of the cornea, which were white large round nodules that resembled sarcoidosis-related granulomatous nodules [3]. The nodules were initially responsive to anti- 
Kitazawa et al.: Diffuse Anterior Retinoblastoma with Sarcoidosis-Like Nodule

inflammatory steroid treatment. However, a biopsy revealed refractory nodules that consisted of undifferentiated cells with rosette formation, indicative of retinoblastoma.

Patients with diffuse infiltrating retinoblastoma tend to be diagnosed at an older age than those with retinoblastoma [4-6]. The clinical features of diffuse infiltrating retinoblastoma are neovascularization, the presence of pseudohypopyon, elevated intraocular pressure, vitreous tumor seeds, subretinal spaces, and vitreous hemorrhage. Shields et al. [5] reported that B-scan ultrasonography imaging is usually negative for nodule calcification and that the tumor presents as poorly defined retinal thickening. However, in our case, there were no obvious retinal findings and there was no calcification inside the nodules. Because of the initial response to steroid treatment, there was a delay in the diagnosis of diffuse anterior retinoblastoma. Diffuse anterior retinoblastoma without retinal involvement is rare, and to our knowledge, there have been only 4 cases reported in the literature [2, 7-10].

Developments in AS-OCT enable it to provide novel findings of ocular tumors with high scanning speed and resolution [11]. In our case, it demonstrated that the white nodules spread horizontally and gradually on the posterior surface of the cornea; in addition, we observed a shrunken iris with nodules, which could have been due to the migration and invasion of tumor cells to vicinal tissues. This suggests that AS-OCT is a useful tool in assessing tumor or inflammatory masses.

Although tumor invasion outside the sclera or to the optic nerve was not observed, the patient underwent chemotherapy after enucleation of the eye. Chemotherapy was necessary because of the delay in diagnosis (over 120 days), which could significantly increase the risk of retinoblastoma metastasis [12]. In addition, tumor invasion to the highly vascularized ciliary body and iris might also increase the risk of metastasis by dissemination of tumor cells.

In this case, a biopsy was necessary to confirm the diagnosis. However, a biopsy could be contraindicated because of the potential for tumor cell dissemination to other tissues, which has been suggested to increase the risk of metastases [13, 14]. Therefore, for diagnostic purposes, a biopsy should be performed by expert ophthalmologists as a last resort.

In conclusion, a differential diagnosis of retinoblastoma should be considered when white nodules refractory to anti-inflammatory therapy occur in the eye, even in the absence of an obvious retinal mass. AS-OCT provided unique findings, which suggested migration and invasion of tumor cells. We would also advise the need for a biopsy to determine the metastatic risk potential.

\section{Acknowledgments}

We thank Dr. Shigenobu Suzuki at the National Cancer Center Hospital for excellent suggestions in terms of the diagnosis and therapy. This study was funded by JSPS KAKENHI Grant Number 26861463.

\section{Statement of Ethics}

Informed consent was obtained from the child's parents for publication of the report and any accompanying images. 
Kitazawa et al.: Diffuse Anterior Retinoblastoma with Sarcoidosis-Like Nodule

\section{Disclosure Statement}

The authors declare that they have no conflict of interest.

\section{References}

1 Young JL, Smith MA, Roffers SD, et al: Retinoblastoma; in Ries LA, Smith MA, Gurney JG, et al. (eds): Cancer Incidence and Survival among Children and Adolescents: United States SEER Program, 1975-1995. Bethesda, National Cancer Institute, 1999, p 73.

-2 Jijelava KP, Grossniklaus HE: Diffuse anterior retinoblastoma: a review. Saudi J Ophthalmol 2013;27:135139.

3 Whitcup SM: Sarcoidosis; in Nussenblatt RB, Whitcup SM (eds): Uveitis, Fundamentals and Clinical Practice, ed 4. St. Louis, Mosby, 2010, pp 278-288.

4 Morgan G: Diffuse infiltrating retinoblastoma. Br J Ophthalmol 1971;55:600-606.

5 Shields CL, Ghassemi F, Tuncer S, et al: Clinical spectrum of diffuse infiltrating retinoblastoma in 34 consecutive eyes. Ophthalmology 2008;115:2253-2258.

6 Schofield PB: Diffuse infiltrating retinoblastoma. Br J Ophthalmol 1960;44:35-41.

7 Grossniklaus HE, Dhaliwal RS, Martin DF: Diffuse anterior retinoblastoma. Retina 1998;18:238-241.

-8 Longmuir SQ, Syed NA, Boldt HC: Diffuse anterior retinoblastoma without retinal involvement. Ophthalmology 2010;117:2034-2038.

-9 Khetan V, Sudrik S, Singh S, et al: Diffuse anterior retinoblastoma with undetectable retinal involvement. J Pediatr Ophthalmol Strabismus 2011;48(Online):e7-e9.

10 Kelly A, Kaufman SC, Ali R, et al: Rare anterior segment retinoblastoma masquerading as corneal endotheliitis. Eye Contact Lens 2015, Epub ahead of print.

11 Medina CA, Plesec T, Singh AD: Optical coherence tomography imaging of ocular and periocular tumours. Br J Ophthalmol 2014;98:40-46.

12 Messmer EP, Heinrich T, Hopping W, et al: Risk factors for metastases in patients with retinoblastoma. Ophthalmology 1991;98:136-141.

13 Shields CL, Manquez ME, Ehya H, et al: Fine-needle aspiration biopsy of iris tumors in 100 consecutive cases: technique and complications. Ophthalmology 2006;113:2080-2086.

14 O'Hara BJ, Ehya H, Shields JA, et al: Fine needle aspiration biopsy in pediatric ophthalmic tumors and pseudotumors. Acta Cytol 1993;37:125-130. 


\section{Case Reports in \\ Ophthalmology}

\begin{tabular}{l|l}
\hline Case Rep Ophthalmol 2015;6:443-447 \\
\hline DOI: 10.1159/000442744 & $\begin{array}{l}\text { c } 2015 \text { The Author(s). Published by S. Karger AG, Basel } \\
\text { www.karger.com/cop }\end{array}$ \\
\hline
\end{tabular}

Kitazawa et al.: Diffuse Anterior Retinoblastoma with Sarcoidosis-Like Nodule
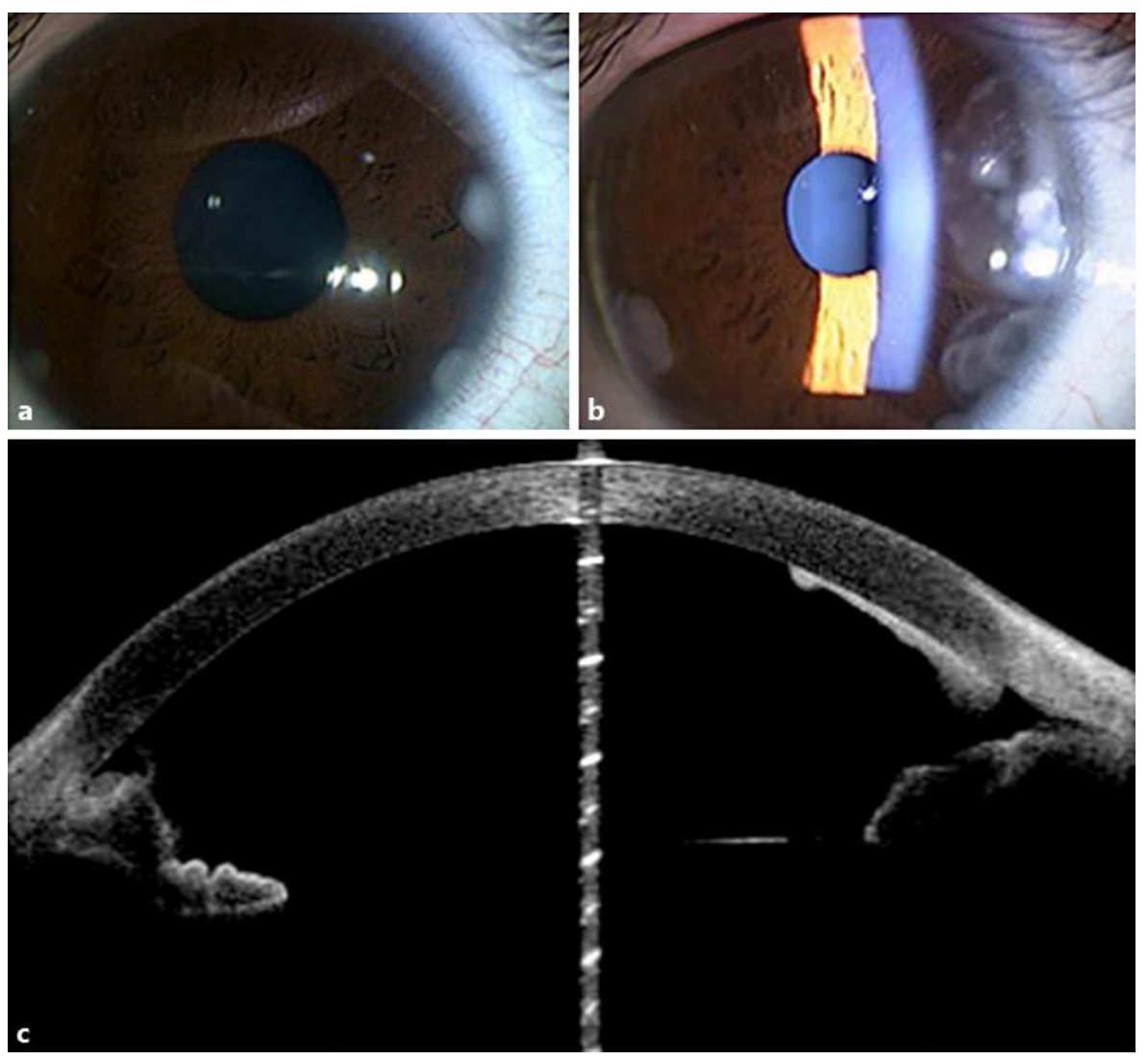

Fig. 1. a Initial slit-lamp examination of the right eye. White nodules are present on the iris and posterior surface of the cornea. b Slit-lamp examination performed prior to the biopsy. Nodules showed horizontal growth across the posterior surface of the cornea. c Image of the anterior segment on optical coherence tomography (SS-100 CASIA, TOMEY, Nagoya, Japan) performed prior to the biopsy.

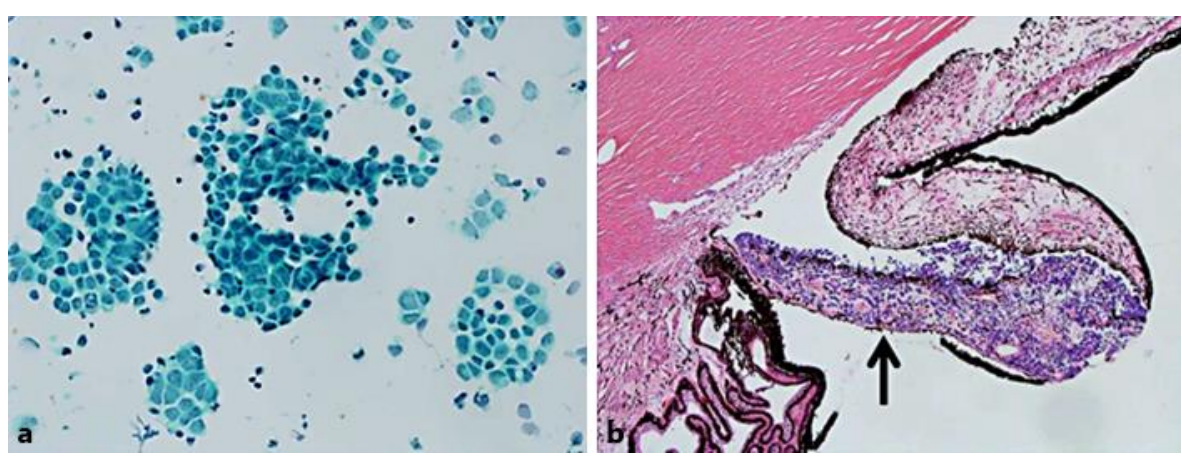

Fig. 2. a The biopsy showed clusters of small undifferentiated cells in a rosette formation. b Tumor cell infiltration in the iris (arrow). 